\title{
EFECTIVIDAD DEL PROGRAMA “MELODÍAS DE CUIDAR”, EN EL MANEJO DE LA ANSIEDAD EN USUARIOS PREOPERATORIOS DEL SERVICIO DE CIRUGÍA DEL HOSPITAL FÉLIX MAYORCA SOTO, TARMA - 2019
}

\author{
Effectiveness of the program "caring melodies", in the management of anxiety in preoperative \\ users of the surgery service of the Hospital Felix Mayorca Soto, Tarma - 2019
}

Isabel María Quispe Montalvo ${ }^{1, b}$, Candy Astrid Cóndor Gomez ${ }^{1, b}$ Nelly Marleni Hinostroza Robles $2, a, c, d$

\footnotetext{
${ }^{1}$ Facultad de Ciencias de la Salud. Escuela Profesional de Enfermería. Universidad Nacional Daniel Alcides Carrión. Tarma, Perú. ${ }^{2}$ Docente de la Facultad de Ciencias de la Salud. Escuela Profesional de Enfermería. Universidad Nacional Daniel Alcides Carrión. Tarma, Perú.

${ }^{a}$ Lic. En Enfermería, ${ }^{b}$ Internas de Enfermería, ${ }^{C}$ Maestro en Enfermería, ${ }^{d}$ Doctora en Ciencias de la Salud
}

\section{RESUMEN}

El estudio tuvo como Objetivo: Determinar el efecto del programa "melodías de cuidar" en el manejo de la ansiedad en usuarios preoperatorios del servicio de cirugía del Hospital Félix Mayorca Soto, Tarma - 2019. Material y métodos: Estudio cuasi experimental, prospectivo, longitudinal y analítico; muestra de 30 usuarios preoperatorios, se aplicó el programa "melodías de cuidar" utilizando una lista de cotejo con pre y postest de Hamilton modificado para evaluar los cambios del nivel de ansiedad. Resultados: El 100\% de usuarios preoperatorios presentan una ansiedad grave antes de aplicar el programa "melodías de cuidar", un 60\% de usuarios migraron a una ansiedad leve luego de participar del programa de musicoterapia pasiva, un $83,3 \%$ de usuarios migraron a una ansiedad leve luego de participar del programa de musicoterapia activa, un $56,7 \%$ de usuarios migraron a una ansiedad leve luego de participar del programa de musicoterapia mixta, un $86,7 \%$ de usuarios preoperatorios disminuyeron su nivel de ansiedad pasando a un nivel leve, y el $13,3 \%$ de usuarios a un nivel moderado. Con la prueba T de student se obtuvo que el valor $P=0,000$ es menor al nivel de significancia 0,05 ; con un $95 \%$ de confianza, la aplicación del programa "melodías del cuidar", mejora significativamente el manejo de la ansiedad en usuarios preoperatorios. Conclusiones: Tras comparar los valores entre el antes y después de aplicar el programa "melodías de cuidar" se observó que ésta disminuye la ansiedad cognitiva, conductual y fisiológica.

Palabras clave: Musicoterapia, ansiedad, preoperatorios, programa "melodías de cuidar"

\section{SUMMARY}

The study was Objective: Determine the effect of the program "caring melodies" in the management of anxiety in preoperative users of the surgery service of the Hospital Félix Mayorca Soto, Tarma - 2019. Material and methods: Quasi-experimental, prospective, longitudinal study and analytical; sample population of 30 preoperative users, the program "melodies of caring" was applied using a quotation list, using a modified Hamilton pre-post-test to evaluate changes in anxiety level. Results: $100 \%$ of preoperative users present severe anxiety before applying the "caring melodies" program, $60 \%$ of users migrated to mild anxiety after participating in the passive music therapy program, $83,3 \%$ of users migrated mild anxiety after participating in the active music therapy program, $56,7 \%$ of users migrated to mild anxiety after participating in the mixed music therapy program, $86,7 \%$ of preoperative users decreased their anxiety level going to a level mild, and $13,3 \%$ of users at a moderate level. With the student's $T$ test, it was obtained that the value $P=0,000$ is less than the 0,05 level of significance; with $95 \%$ confidence, the application of the program "caring melodies", significantly the management of anxiety in preoperative users. Conclusions: After comparing the values between before and after applying the "caring melodies" program, this decrease in cognitive, behavioral and physiological anxiety.

Key words: Music therapy, anxiety, preoperative, "caring melodies" program. 


\section{INTRODUCCIÓN.}

La enfermería abarca un campo holístico e integral en el cuidado, considera el aspecto emocional y mental del usuario, que en muchas ocasiones se deja de lado priorizando solo la respuesta biológica, mediante la aplicación de la musicoterapia se generará un ambiente más tranquilo y libre de expresar emociones, esto aporta muchos beneficios siendo uno de ellos el manejo de la ansiedad; estando presente en todo usuario preoperatorio, solo difieren en el nivel en que se manifiestan ya sea leve, moderada o grave (1).

En la actualidad la medicina alternativa no requiere muchos gastos para poder utilizarlos, entre ellos están: musicoterapia, acupuntura, digitopuntura, meditación, risoterapia, yoga, entre otros. Siendo la musicoterapia, hoy en día, reconocida como una disciplina científica y forma parte de una de las acciones complementarias de la Organización Mundial de la Salud (OMS), reconociéndola como una gran alternativa terapéutica, manifestando que se trata de un tipo de tratamiento sin efectos secundarios dañinos y es muy económico y brinda beneficios en la salud - enfermedad, por lo que consideramos trascendente en el cuidado de enfermería (2).

Anchudia R, Aragundi $H$. en Guayaquil, 2015; realizaron un estudio descriptivo transversal a 256 usuarios con el objetivo de evaluar los efectos de la musicoterapia en la disminución del nivel de ansiedad y dolor en las fases pre y post operatorio de pacientes adultos del Hospital Universitario., evidenciándose que la mayor cantidad de pacientes no presentan ningún tipo de ansiedad, mientras que una minoría presentó un estado grave de ansiedad y depresión, previo a la sesión de Musicoterapia, siendo los síntomas somáticos, gastrointestinales genitourinarios los principales que resaltaban en los pacientes(3).
Khosravy H, Lima 2017, realizó un estudio de nivel aplicativo, tipo cuasi experimental, método descriptivo de corte trasversal, con 30 usuarios tuvo como objetivo determinar la efectividad de la musicoterapia como cuidado de enfermería frente a la ansiedad del paciente en el periodo preoperatorio inmediato, en donde obtuvo como conclusión que la musicoterapia es efectiva como cuidado de enfermería al paciente frente a la ansiedad preoperatoria inmediata, mediante la afirmación de la hipótesis alterna comprobada a través la prueba de $\mathrm{T}$ de student arrojando un $\mathrm{t}=16,41$ (4).

Jiménez A. Lambayeque 2016, realizó un estudio de tipo cuantitativo experimental, prospectiva, transversal y analítica, en un total de 88 usuarios pediátricos entre 6 y 9 años, tuvo como objetivo evaluar la influencia de la musicoterapia para el manejo de ansiedad en pacientes pediátricos atendidos en la clínica de estomatología. Obteniendo la siguiente conclusión: La musicoterapia influye de manera positiva en el manejo de ansiedad, que presentan los pacientes pediátricos durante los tratamientos en la clínica de estomatología de la Universidad Señor de Sipán (5).

Otra investigación es la de Llaccho M, Nuñez $\mathrm{N}$, Pachas D. Lima 2014, quienes realizaron un estudio experimental de tipo cuantitativo, transversal, siendo su población objetivo todos los usuarios que acuden al servicio de cirugía menor con programación para cirugía de hernia inguinal, con un promedio de 120 usuarios de 9 a 12 años que cumplan los criterios de selección, la investigación señalo como objetivo determinar la efectividad de la musicoterapia en el nivel de ansiedad de los usuarios de 9 a 12 años durante el pre operatorio de Hernia inguinal en el Instituto de Salud del Niño, el estudio surgió debido a la falta de difusión de tratamientos alternativos no invasivos para el manejo de usuarios y la falta de experiencia en el tema de muchos profesionales, llegando a la conclusión que la musicoterapia es una alternativa de relajación que disminuye 
eficazmente la ansiedad en los niños, desviando su atención para facilitar los procedimientos preventivos; haciéndolos sencillos, agradables y de corta duración (6).

En las prácticas clínicas realizadas en el servicio de cirugía del Hospital Félix Mayorca Soto, en el año 2018, se logró observar que los usuarios preoperatorios presentaban mucha incomodidad propia del dolor y tensión, como a la vez preocupación y temor a la muerte, a menudo no pueden conciliar el sueño y presentan nerviosismo; dando por consecuencia cansancio, malestar ansiedad e incremento de la presión arterial a unas horas de la operación, ello reduce la recuperación rápida del paciente. El estudio es un aporte para enriquecer la práctica profesional de enfermería, implementar una técnica ya existente que no tenía mucha relevancia.

Tomando en consideración el campo holístico de enfermería se desarrolló el programa: "melodías de cuidar" con el objetivo de determinar el efecto que este tiene en el manejo de la ansiedad de los usuarios preoperatorios en el servicio de cirugía del Hospital Félix Mayorca Soto, Tarma.

\section{MATERIALES Y MÉTODOS.}

El diseño de la investigación es de enfoque cuantitativo, de tipo cuasi experimental porque es de muestra no aleatoria, analítico, prospectivo ya que se utilizó los datos generados por nuestro instrumento de medición y longitudinal porque se realizó dos mediciones: el antes y el después para luego ser comparados. La población muestral fue de 30 usuarios pre operatorios del servicio de cirugía del Hospital Félix Mayorca Soto, entre los meses de junio y septiembre del año 2019.

Se creó un programa de musicoterapia denominado "melodías de cuidar", considerando las siguientes dimensiones: musicoterapia pasiva, activa y mixta, para su ejecución se utilizó dispositivos de reproducción sonora, siendo emitidos con un volumen adecuado sin sobrepasar los 55 decibeles, las músicas transmitidas eran respetando la preferencia del usuario. Para la evaluación del cumplimiento de las actividades del programa se realizó una lista de cotejo.

La elaboración del instrumento que mide el nivel de ansiedad, tuvo como base el Test del psiquiatra Max Hamilton (7), siendo modificado según las manifestaciones de ansiedad observadas en el usuario preoperatorio. Este test consideró tres dimensiones: cognitivo, conductual y fisiológico, y 12 ítems en donde el investigador cuestiono al usuario quien respondió en base a los signos y síntomas que presenta dando como respuesta a tres alternativas: si, a veces, no.

Se registró los datos obtenidos de la aplicación del pre test de Hamilton modificado, seguidamente el usuario participo del programa de musicoterapia "melodías de cuidar" en sus diferentes dimensiones pasiva, activa y mixta; luego se realizó el postest de Hamilton modificado, en donde se registró las respuestas que dan los usuarios pre operatorios.

Teniendo las encuestas realizadas de 30 usuarios preoperatorios, se lleva a cabo el procesamiento y vaciado de datos en los programas de: Excel 2016, SPSS versión 25 y Minitab 18, se utilizó el programa Excel para la elaboración de cuadros estadísticos luego se realizó el análisis y discusión de los resultados obtenidos, por último, en el programa Minitab 18 se realizó la prueba de hipótesis utilizando la técnica $T$ de student de una muestra.

\section{RESULTADOS.}

Se utilizó como población muestral a 30 usuarios pre operatorios que cuenten con los siguientes criterios: Personas entre los 21 a 60 años con operaciones programadas, usuarios sin discapacidad auditiva y con disposición de ser parte del estudio. 
En la Tabla 1 se observa que los 30 encuestados siendo un $100 \%$ presentan un nivel de ansiedad grave antes de la operación en el servicio de cirugía del Hospital Félix Mayorca Soto. Con la prueba $T$ de student se obtuvo que el valor de probabilidad $\mathrm{P}=0,000$ es menor que el nivel de significancia $\alpha=0,05$; se rechaza la hipótesis nula y se acepta la hipótesis de investigación. Esto nos permite concluir para un $95 \%$ de confianza que, los usuarios preoperatorios presentan en su totalidad el nivel de ansiedad grave en el servicio de cirugía del Hospital Félix Mayorca Soto Tarma.

En la Tabla 2 los resultados que se muestran indican claramente que, 18 usuarios preoperatorios han migrado hacia los niveles de ansiedad leve en su dimensión cognitiva después de haber participado del programa de musicoterapia pasiva. En la tabla se evidencia los ítems considerados en el instrumento. Con la prueba $T$ de student se obtuvo que el valor de probabilidad $P=0,000$ es menor que el nivel de significancia $\alpha=$ 0,05 ; se rechaza la hipótesis nula y se acepta la hipótesis de investigación. Esto nos permite concluir para un $95 \%$ de confianza que, con la aplicación del programa de musicoterapia "melodías de cuidar" en su dimensión pasiva mejora significativamente el manejo de la ansiedad cognitiva en usuarios preoperatorios del servicio de cirugía del Hospital Félix Mayorca Soto - Tarma.

En la Tabla 3 se muestra que 25 usuarios preoperatorios han migrado hacia los niveles de ansiedad leve en la dimensión conductual después de haber participado el programa de musicoterapia activa. En la tabla se evidencia los ítems considerados en el instrumento. Con la prueba $\mathrm{T}$ de student se obtuvo que el valor de probabilidad $\mathrm{P}=0,000$ es menor que el nivel de significancia $\alpha=$ 0,05 ; se rechaza la hipótesis nula y se acepta la hipótesis de investigación. Esto nos permite concluir para un $95 \%$ de confianza que, con la aplicación del programa de musicoterapia "melodías de cuidar" en su dimensión activa mejora significativamente el manejo de la ansiedad conductual en usuarios preoperatorios del servicio de cirugía del Hospital Félix Mayorca Soto - Tarma.

En la Tabla 4 se evidencia que 17 usuarios preoperatorios han migrado hacia los niveles de ansiedad leve en su dimensión fisiológica después de haber participado del programa de musicoterapia mixta. En la tabla se evidencia los ítems considerados en el instrumento. Con la prueba $T$ de student se obtuvo que el valor de probabilidad $P=0,000$ es menor que el nivel de significancia $\alpha=$ 0,05 ; se rechaza la hipótesis nula y se acepta la hipótesis de investigación. Esto nos permite concluir para un $95 \%$ de confianza que, con la aplicación del programa de musicoterapia "melodías de cuidar" en su dimensión mixta mejora significativamente el manejo de la ansiedad fisiológica en usuarios preoperatorios del servicio de cirugía del Hospital Félix Mayorca Soto - Tarma.

En la Tabla 5 se indican claramente que los 26 encuestados presentan un nivel de ansiedad leve antes de la operación en el servicio de cirugía y luego de la ejecución del programa de musicoterapia "melodías de cuidar" del Hospital Félix Mayorca Soto, Tarma. Con la prueba $T$ de student se obtuvo que el valor de probabilidad $P=0,000$ es menor que el nivel de significancia $\alpha=0,05$; se rechaza la hipótesis nula y se acepta la hipótesis de investigación. Esto nos permite concluir para un $95 \%$ de confianza que, los usuarios preoperatorios presentan en su gran mayoría el grado leve de ansiedad en el servicio de cirugía del Hospital Félix Mayorca Soto, luego del desarrollo del programa de musicoterapia "melodías de cuidar", Tarma.

En la Tabla 6 los resultados que se muestran indican claramente que, 26 usuarios preoperatorios disminuyeron su nivel de ansiedad, pasando a un nivel leve y 4 usuarios a un nivel moderado. Con la prueba $\mathrm{T}$ de student se obtuvo que el valor de 
probabilidad $\mathrm{P}=0,000$ es menor que el nivel de significancia $\alpha=0,05$; se rechaza la hipótesis nula y se acepta la hipótesis de investigación. Esto nos permite concluir para un $95 \%$ de confianza que, con la aplicación del programa de musicoterapia "melodías de cuidar" se mejora significativamente el manejo de la ansiedad en usuarios preoperatorios del servicio de cirugía del Hospital Félix Mayorca Soto - Tarma.

Tabla 1. Nivel de ansiedad en usuarios preoperatorios del servicio de cirugía antes de la aplicación del programa "melodías de cuidar" en el Hospital Félix Mayorca Soto - 2019

\begin{tabular}{lcc}
\multicolumn{1}{c}{ ANSIEDAD } & PRETEST & $\%$ \\
A: Grave & 30 & $100 \%$ \\
\hline B: Moderada & 0 & $0 \%$ \\
\hline C: Leve & 0 & $0 \%$ \\
\hline TOTAL & $\mathbf{3 0}$ & $\mathbf{1 0 0} \%$ \\
Fuente: Base de datos &
\end{tabular}

Tabla 2. Nivel de ansiedad cognitivo en usuarios preoperatorios antes y después de la aplicación del programa de musicoterapia pasiva en el Hospital Félix Mayorca Soto - 2019

\begin{tabular}{|c|c|c|c|c|c|}
\hline ÍTEMS EVALUADOS & ANSIEDAD & PRETEST & $\%$ & POSTEST & $\%$ \\
\hline 1. ¿Tiene miedo e ideas negativas para el & A: Grave & 30 & $100 \%$ & 0 & $0 \%$ \\
\hline $\begin{array}{l}\text { 2. ¿Desea que la operación se le realice lo } \\
\text { más pronto posible? }\end{array}$ & B: Moderada & 0 & $0 \%$ & 12 & $40 \%$ \\
\hline $\begin{array}{l}\text { ¿Se encuentra tenso y presiente que algo } \\
\text { malo va ocurrir? }\end{array}$ & C: Leve & 0 & $0 \%$ & 18 & $60 \%$ \\
\hline salud? & TOTAL & 30 & $100 \%$ & 30 & $100 \%$ \\
\hline
\end{tabular}

Fuente: Base de datos

Tabla 3. Nivel de ansiedad conductual en usuarios preoperatorios antes y después de la aplicación del programa de musicoterapia activa en el Hospital Félix Mayorca Soto - 2019

\section{\begin{tabular}{l|lllll} 
ÍtEMS EVALUADOS & ANSIEDAD & PRETEST & $\%$ & POSTEST & $\%$
\end{tabular}}

\begin{tabular}{|c|c|c|c|c|c|}
\hline $\begin{array}{l}\text { 5. ¿Está triste la mayor parte del } \\
\text { tiempo? }\end{array}$ & A: Grave & 28 & $93,3 \%$ & 2 & $6,7 \%$ \\
\hline $\begin{array}{l}\text { 6. ¿Se siente intranquilo, no puede } \\
\text { estar quieto, se retuerce las manos, } \\
\text { se muerde las uñas o los labios? }\end{array}$ & B: Moderada & 2 & $6,7 \%$ & 3 & $10 \%$ \\
\hline $\begin{array}{l}\text { 7. ¿Se despierta con gran facilidad } \\
\text { y le dificulta conciliar el sueño otra } \\
\text { vez? } \\
\text { 8. ¿Le cuesta mucho relajarse y }\end{array}$ & C: Leve & 0 & $0 \%$ & 25 & $83,3 \%$ \\
\hline siente mucha tensión? & TOTAL & 30 & $100 \%$ & 30 & $100 \%$ \\
\hline
\end{tabular}

Fuente: Base de datos 
Tabla 4. Nivel de ansiedad fisiológica en usuarios preoperatorios antes y después de la aplicación del programa de musicoterapia mixta en el Hospital Félix Mayorca Soto - 2019

\begin{tabular}{|c|c|c|c|c|c|}
\hline ÍTEMS EVALUADOS & ANSIEDAD & PRETEST & $\%$ & POSTEST & $\%$ \\
\hline \multirow{4}{*}{$\begin{array}{l}\text { 9. ¿Estaba el paciente tenso, nervioso, } \\
\text { agitado, impaciente, tembloroso, pálido, } \\
\text { hiperventilando o transpirando? } \\
\text { 10. ¿Tiende a sudar mucho? } \\
\text { 11. ¿Tiene taquicardia, palpitaciones, } \\
\text { opresión, dolor torácico, latido de venas } \\
\text { y arterias y sensación de desmayo? } \\
\text { 12. ¿Siente mucha rigidez e } \\
\text { hipersensibilidad? }\end{array}$} & A: Grave & 27 & $90 \%$ & 0 & $0 \%$ \\
\hline & B: Moderada & 3 & $10 \%$ & 13 & $43,3 \%$ \\
\hline & C: Leve & 0 & $0 \%$ & 17 & $56,7 \%$ \\
\hline & TOTAL & 30 & $100 \%$ & 30 & $100 \%$ \\
\hline
\end{tabular}

Fuente: Base de datos

Tabla 5. Nivel de ansiedad en usuarios preoperatorios del servicio de cirugía, luego de la aplicación del programa de musicoterapia "melodías de cuidar" en el Hospital Félix Mayorca Soto - 2019

\begin{tabular}{lcc} 
ANSIEDAD & POSTEST & $\%$ \\
\hline A: Grave & 0 & $0 \%$ \\
\hline B: Moderada & 4 & $13,3 \%$ \\
\hline C: Leve & 26 & $86,7 \%$ \\
\hline TOTAL & 30 & $100 \%$ \\
\hline
\end{tabular}

Fuente: Base de datos

Tabla 6. Nivel de ansiedad en usuarios preoperatorios antes y después de la aplicación del programa de musicoterapia "Melodías de cuidar" Hospital Félix Mayorca Soto - 2019

\begin{tabular}{lcccc}
\multicolumn{1}{c}{ ANSIEDAD } & PRETEST & $\%$ & POSTEST & $\%$ \\
\hline A: Grave & 30 & $100 \%$ & 0 & $0 \%$ \\
\hline B: Moderada & 0 & $0 \%$ & 4 & $13,3 \%$ \\
\hline C: Leve & 0 & $0 \%$ & 26 & $86,7 \%$ \\
\hline \multicolumn{1}{c}{ TOTAL } & 30 & $100 \%$ & 30 & $100 \%$ \\
\hline
\end{tabular}

Fuente: Base de datos

\section{DISCUSIÓN.}

La intervención de enfermería en el periodo preoperatorio tiene el objetivo de reducir los factores que generan tensión y las condiciones adversas que afectan el buen funcionamiento del organismo con el fin de proporcionar apoyo emocional y/o utilizar estrategias para afrontar tal situación estresante como lo es una intervención quirúrgica. La musicoterapia al ser considerado como un tratamiento complementario con grandes evidencias demostradas desde tiempos ancestrales brinda a la enfermera una herramienta para reducir la ansiedad en el paciente en la etapa preoperatoria; sin conllevar riesgos al paciente al ser un agente neuroprotector natural tal como lo menciona Juan Orjuela Rojas doctor de Psiquiatría de la Pontificia Universidad Javeriana de Colombia (8).Por ello conocer la efectividad que tiene la musicoterapia sobre la ansiedad durante la 
etapa preoperatoria resulta de importancia en el cuidado enfermero para que el usuario logre afrontar este momento difícil y se convierta en un proceso más afrontable, en el estudio se demostró que el 100\% (30 de los pacientes) presentaban un nivel de ansiedad preoperatoria grave, porcentaje que se disminuye al $0 \%$ ósea ningún paciente, en este nivel de ansiedad luego de la aplicación del programa de musicoterapia "melodías de cuidar", se observó que un $86,6 \%$ (26 pacientes) presentaba un nivel de ansiedad leve y $13,4 \%$ (4 pacientes) presentaba un nivel de ansiedad moderado.

Esto podría indicar que el usuario ingrese mucho más relajado a ser intervenido quirúrgicamente gracias a la musicoterapia, este resultado concuerda con el obtenido por Crispín Gigante el cual concluye que la ansiedad se puede disminuir con la música además del cortisol, produciendo que el paciente se siente relajado y tranquilo (9), para así evitar efectos negativos de la ansiedad y la amenaza de una recuperación lenta en la etapa postoperatoria.

La ansiedad es descrita como el sentimiento desagradable de temor que se percibe como señal de alarma ante un peligro amenazante en su mayoría desconocida. Peter Lang en el año 1968 propone su teoría de la tridimensionalidad de la ansiedad (cognitiva, fisiológica y conductual) (10). Por ello conocer específicamente los efectos de la musicoterapia sobre estas dimensiones constituye un indicador importante. En el estudio se encontró que 100\% (30 pacientes) de la población presentaba un nivel de ansiedad grave en la dimensión cognitiva cifra que se redujo a ansiedad leve en un $60 \%$ (18 pacientes) luego de ser aplicada la musicoterapia pasiva, es decir este porcentaje nos revela que muchos de sus sentimientos de preocupación, temor, inseguridad y pensamientos negativos producto de la ansiedad que genera una intervención quirúrgica fueron disminuidos gracias a la musicoterapia pasiva, lo cual concuerda con el estudio de Lliner Soplin Mendoza, en el año 2015, quien reporta que la musicoterapia produce pensamientos positivos a la población produciendo una relajación en la parte cognitiva luego de ser aplicada la musicoterapia(11). En la dimensión fisiológica se observó que la ansiedad de nivel grave presentada antes la musicoterapia era de 90\% (27 pacientes) luego de aplicar la musicoterapia mixta este porcentaje paso a ser $56,6 \%$ (17 pacientes) lo cual indica que las manifestaciones de ansiedad como taquicardia, sudoración, palpitaciones 0 dolores torácicos se redujeron. En la dimensión conductual se observó que del 93,3\% (28 pacientes) de ansiedad grave que presentaban los pacientes paso a ser el $83,3 \%$ (25 pacientes) es decir casi en su totalidad presentaban ansiedad leve en esa dimensión luego de la aplicación de la musicoterapia activa, en estas cifras podemos observar que a pesar que los usuarios presentaban muchos malestares de hiperactividad, movimientos repetitivos, entre otros; estos comportamientos observables pudieron disminuir gracias a la musicoterapia activa.

\section{CONCLUSIONES.}

1. El programa de musicoterapia "melodías de cuidar" es efectiva para el manejo de la ansiedad en pacientes pre operatorios, mediante la afirmación de la hipótesis alterna comprobada a través la prueba de $\mathrm{T}$ de student de una muestra arrojando un $\mathrm{p}=0,000$

2. La ansiedad antes de la aplicación de la musicoterapia resulto ser de nivel grave en su totalidad de las 30 personas encuestadas.

3. Dentro de los datos más relevantes, demostrando cifras considerables fue el impacto que tuvo el programa de musicoterapia "melodías de cuidar" en su dimensión activa frente a la ansiedad conductual.

4. Tras comparar los valores entre el antes y después de la aplicación del programa de musicoterapia "melodías de cuidar" se observó que esta disminuye la ansiedad en sus tres dimensiones tanto la cognitiva, conductual y fisiológica. 


\section{AGRADECIMIENTOS.}

A la asesora por sus sabias enseñanzas que sirvieron de guía en el proceso investigativo. A la comisión científica evaluadora del Congreso Nacional de Estudiantes de Enfermería de Cuzco Unsaac por permitirnos ser partícipes y ganadores del concurso de investigación en su modalidad exposición oral con el proyecto (Efectividad del programa "melodías de cuidar", en el manejo de la ansiedad en usuarios preoperatorios del servicio de cirugía del Hospital Félix Mayorca Soto, Tarma - 2019).

\section{CORRESPONDENCIA.}

Isabel María Quispe Montalvo

Correo electrónico: iquispem.799@gmail.com

\section{REFERENCIAS BIBLIOGRÁFICAS.}

\section{Quintero A, Yasno D, Riveros $O$,} Castillo J, Borráez B. Ansiedad en paciente prequirúrgico: problema que nos afecta a todos. Rev. Colombia Cir. $2017 ; 32: 115-20$

2. Somos Pacientes. La comunidad de asociaciones de pacientes. La musicoterapia se implanta como terapia complementaria en Extremadura (Internet). España: Somos pacientes; 2015. (Citado el 8 de Noviembre 2019). Disponible en: https://www.somos pacientes.com/noticias/sanidad/lamusicoterapia-se-implanta-comoterapia-complementaria-enextremadura/

3. Anchudia R, Aragundi L. Disminución del nivel de ansiedad y dolor con musicoterapia en fases pre y postoperatorio pacientes adultos hospital universitario enero a marzo del 2015. Tesis licenciatura. Guayaquil, Ecuador. Universidad de Guayaquil; 2015.92 pp.
4. Khosravy H. Efectividad de la musicoterapia como cuidado de enfermería sobre ansiedad en pacientes en etapa preoperatoria en clínica privada. Tesis de especialidad. Lima, Perú. Facultad de Medicina, Segunda Especialización Enfermería, Universidad Nacional Mayor San Marcos; 2017.92 pp.

5. Jiménez A. Influencia de la musicoterapia para el manejo de ansiedad en pacientes pediátricos atendidos en la clínica de estomatología de la universidad Señor de Sipán. Tesis de título. Pimentel, Perú. Universidad Señor de Sipán; 2016. 74 pp.

6. Llaccho $M$, Nuñez $M$, Pachas $D$. Efectividad de la musicoterapia en el nivel de ansiedad de los pacientes de 9 a 12 años durante el pre operatorio de hernia inguinal. Tesis de segunda especialidad. Lima, Perú: Universidad Peruana Cayetano Heredia; 2014. 45 pp.

7. Sabater V. La escala de ansiedad de Hamilton. Rev la mente es maravillosa (Internet) 2018. (Citado el 23 de setiembre 2020) Disponible en: https://lamenteesmaravillosa.com/laescala-de-ansiedad-de-hamilton/.

8. Rojas M, Azevedo D. Estrategia de enfermería para disminuir los niveles de ansiedad en pacientes sometidos a intervención quirúrgica en el Hospital Universitario Antonio Patricio de Alcalá, Cumana, Venezuela. Rev Multidisciplinaria del Consejo de Investigación de la Universidad de Oriente. 2013; 25(3):273 - 278.

9. Gigante C. Efectos de la musicoterapia receptiva sobre la ansiedad y el estrés (cortisol) en una población joven universitaria. Alcalá, España. Universidad de Alcalá; 2011.71 pp. 
10. Aragón R. Modelo tridimensional de la ansiedad propuesto por Peter Lang. Rev psiqueviva El Poder de la Mente (Internet). España; 2017 (Citado el 23 de setiembre 2020) Disponible en: https://psiqueviva.com/modelotridimensional-de-la-ansiedadpropuesto-por-peter-lang/.
11. Soplín L. Efectos de la musicoterapia sobre el estado de ánimo de los adultos mayores en el Hospital Regional Virgen de Fátima. Chachapoyas, Perú. Universidad Nacional Toribio Rodríguez de Mendoza de Amazonas; 2015.

Recibido: 27/08/2020

Aprobado para publicación: 26/11/2020 